Revue européenne des sciences sociales

European Journal of Social Sciences

XL-124 | 2002

Histoire, philosophie et sociologie des sciences

Identité personnelle et logique du social

Razmig Keucheyan

(2) OpenEdition

Journals

Édition électronique

URL : http://journals.openedition.org/ress/592

DOI : $10.4000 /$ ress.592

ISSN : 1663-4446

Éditeur

Librairie Droz

Édition imprimée

Date de publication : 1 août 2002

Pagination : 263-282

ISBN : 2-600-00806-3

ISSN : 0048-8046

Référence électronique

Razmig Keucheyan, «Identité personnelle et logique du social », Revue européenne des sciences sociales [En ligne], XL-124 | 2002, mis en ligne le 01 décembre 2009, consulté le 02 mai 2019. URL http://journals.openedition.org/ress/592; DOI : 10.4000/ress.592

(c) Librairie Droz 
Razmig KEUCHEYAN

\section{IDENTITÉ PERSONNELLE ET LOGIQUE DU SOCIAL}

Le concept d'identité est très en vogue depuis quelques temps. Nombreux sont les sociologues qui le mettent à profit pour expliquer les croyances et les comportements des acteurs sociaux. Ce concept fait aussi l'objet d'un usage abondant dans le langage courant, alors que la presse l'exploite régulièrement pour rendre compte de l'actualité. Le problème est que la plupart du temps, ses applications sont vagues. Il existe un décalage profond entre la facilité avec laquelle cette notion est utilisée et la difficulté de la définir de façon rigoureuse.

Le motif principal de ce décalage est à trouver dans le caractère polysémique de ce concept. L'identité est parfois synonyme de «personnalité » ou de «subjectivité », et désigne alors les traits de caractère d'un ou de plusieurs individus. Mais il s'agit aussi d'un concept formel, qui a trait au rapport qu'entretient une entité (humaine ou non) avec elle-même au cours de son existence. Enfin, l'identité a été l'objet de nombreux usages sociologiques, certains paradigmes classiques de la science sociale en ont même fait le cœur de leur analyse de la réalité sociale.

La présente étude a pour ambition d'éclaircir quelque peu les enjeux liés à l'utilisation du concept d'identité en sciences sociales. Pour ce faire, nous en distinguerons trois conceptions sociologiques. Nous montrerons que ces conceptions manquent soit de cohérence interne, soit de congruence avec la réalité. Dans un second temps, nous effectuerons un détour par le débat philosophique portant sur l'identité. Nous nous attarderons, en particulier, sur la théorie de l'identité personnelle de Derek Parfit, la plus convaincante à l'heure actuelle. Dans un troisième temps, nous reviendrons aux sciences sociales pour montrer que malgré tous les problèmes qu'elle pose, la notion d'identité peut et doit être conservée comme catégorie d'analyse fondamentale de la sociologie.

\section{LE CONCEPT D'IDENTITÉ EN SCIENCES SOCIALES}

On peut distinguer trois conceptions de l'identité dans les sciences sociales: l'identité archétypale, l'identité plurielle et l'identité construite. Ces conceptions sont évidemment idéales-typiques, mais recoupent largement les usages effectifs de cette notion.

\section{L'identité archétypale}

La conception archétypale de l'identité repose sur le raisonnement suivant:

1. les groupes sociaux existent; 
2. ils ont une identité à part entière;

3. cette identité se transmet à chaque individu qui les compose;

4. l'identité personnelle est le «reflet» de l'identité du groupe dans lequel l'individu évolue.

La conception archétypale présuppose une ontologie sociale holiste. Elle affirme que les groupes sociaux existent, au même titre que les autres objets qui composent la réalité, et que cette existence leur confère une identité. Elle implique également que chaque acteur social «porte» l'identité du groupe auquel il appartient. Plus précisément, l'individu ne dispose d'une identité que pour autant qu'il évolue dans une communauté. Par conséquent, les identités personnelles sont les «reflets » de l'identité collective.

La conception archétypale de l'identité apparaît dans l'un des paradigmes classiques de la science sociale: le culturalisme. Les sociologues culturalistes insistent sur le rôle décisif joué par des facteurs d'ordre culturel dans la formation des identités personnelles. Selon eux, l'identité d'un individu se réduit, pour l'essentiel, à l'identité de la société dans laquelle il vit. De ce point de vue, on peut dire qu'il n'existe pas à proprement parler d'identité personnelle. Il n'est que des identités sociales ou culturelles.

Certains passages de l'œuvre d'Abram Kardiner illustrent bien cette idée ${ }^{1}$. Kardiner, on le sait, est à l'origine de la distinction entre les institutions primaires et les institutions secondaires. Les premières regroupent les institutions que l'individu rencontre au début de sa vie, au nombre desquelles on compte par exemple la famille. Les institutions secondaires, quant à elles, sont plus tardives, et incluent notamment l'école et la religion.

La notion d'institution primaire a inspiré à Kardiner le concept de personnalité de base, qui désigne l'ensemble des traits psychologiques dont un individu hérite de la société dans laquelle il évolue. Ces traits ont deux caractéristiques principales. D'une part, comme les institutions primaires, ils s'imposent à tous les membres de la société considérée. Tout individu exemplifie la personnalité de base de la société dans laquelle il vit. D'autre part, la personnalité de base joue un rôle déterminant quant aux croyances et aux comportements que sont susceptibles d'adopter les acteurs sociaux.

Le concept de personnalité de base correspond bien à la définition que nous avons donnée de l'identité archétypale. Kardiner prend pour point de départ une ontologie sociale holiste. Les groupes sociaux existent et disposent d'une identité, laquelle recouvre les traits culturels qui les distinguent d'autres entités sociales. L'anthropologue soutient ensuite que l'identité d'un groupe s'impose à l'ensemble de ses membres, par l'entremise justement de la personnalité de base. L'identité d'une personne est donc le «reflet» de l'identité du collectif auquel elle appartient.

La conception archétypale de l'identité a fait l'objet de nombreuses critiques. La plus évidente est qu'elle ne rend pas compte de la complexité des rapports sociaux dans les sociétés modernes. Au sein de ces dernières, les acteurs sociaux sont l'objet d'une pluralité d'influences, qui résultent de leurs nombreuses inser-

Abram Kardiner, 1969, L'individu dans sa société, Paris, Gallimard. 
tions sociales. Partant, soutenir qu'un unique facteur détermine complètement, ou même principalement, l'identité d'une personne semble incohérent. Certes, il est possible que certains - ou l'un - des groupes auxquels appartient un individu influent plus fortement sur son identité. Par exemple, il est plausible d'affirmer que l'appartenance au groupe ouvrier dans les années 1970 primait sur toutes les autres. Mais, d'une part, il est peu probable qu'une telle appartenance détermine la personnalité des acteurs sociaux au point de faire d'eux les porteurs d'une seule identité. D'autre part, comme l'ont démontré Beaud et Pialoux dans le cas des ouvriers $^{2}$, la dynamique des sociétés contemporaines est telle que même les identités les plus fermement établies peuvent entrer en crise.

\section{L'identité plurielle}

Ces considérations nous conduisent à envisager une seconde conception de l'identité: l'identité plurielle. Cette dernière reprend à son compte les quatre éléments qui définissent l'identité archétypale, mais en ajoute un cinquième:

5. tout individu appartient à plusieurs groupes sociaux, dont chacun lui confère une identité. Tout individu dispose donc de plusieurs identités.

La conception plurielle de l'identité est très répandue à l'heure actuelle. Selon l'un de ses partisans, Alain Caillé, tout acteur social relève d'au moins trois «zones d'appartenance ${ }^{3}$. La première est composée des « réseaux de sociabilité primaire», qui regroupent la famille ou l'entreprise dans laquelle il travaille. Une seconde zone est le «macrosujet collectif», qui désigne l'ensemble des communautés religieuses, ethniques ou politiques auxquelles il se sent appartenir. Même si ces «macrosujets » sont éloignés de l'expérience directe de l'individu, ils peuvent avoir une grande influence sur ses croyances et ses actions. La troisième zone d'appartenance est l' «humanité entière». Selon Caillé, à l'heure de la «mondialisation », le fait de nous considérer comme membre de l'humanité inspire bon nombre de nos comportements.

A chacune de ces zones d'appartenance correspond une identité, ce qui fait des acteurs sociaux des êtres «pluri-identitaires ». Les réseaux de sociabilité primaire influent sur notre manière de parler, ou notre rapport au travail. Mais ils ne suffisent pas à rendre compte de certaines de nos attitudes, qui les transcendent manifestement. Ainsi, pour comprendre pourquoi un individu est tenté de devenir un terroriste islamiste, il faut faire appel à une échelle d' «identification» supérieure, à savoir qu'il appartient à une communauté de croyants dont il considère que les droits sont bafoués. En somme, chacun des cercles auxquels nous appartenons nous confère une identité différente, susceptible de se superposer aux autres et même parfois d'entrer en conflit avec elles.

La conception plurielle de l'identité cherche à dépasser le défaut principal de l'identité archétypale, à savoir sa détermination par un unique groupe social. Le

2 Stéphane Beaud et Michel Pialoux, 1999, Retour sur la condition ouvrière. Enquête dans les usines Peugeot de Sochaux-Montbéliard, Paris, Fayard.

3 Cité par Jean-François Dortier, «L'individu dispersé et ses identités multiples », in Jean-Claude Ruano-Borbalan (éd.), 1998, L’identité, Paris, Ed. Sciences Humaines, pp. 52-53. 
problème est que ce dépassement s'effectue au prix d'un contresens évident. On distingue traditionnellement entre deux formes d'identité: l'identité numérique et l'identité qualitative. La première exprime le rapport qu'entretient un objet avec lui-même tout au long de son existence. Par exemple, un arbre de deux ans est numériquement identique au même arbre cinq ans plus tard, même si sa composition matérielle a changé. La seconde désigne le degré maximal de ressemblance entre deux entités. Elle caractérise par exemple la relation existant entre deux jumeaux homozygotes, ou entre deux «moments» du même arbre.

Les partisans de la conception plurielle de l'identité ne font certainement pas référence à l'identité numérique, puisqu'une entité qui aurait plusieurs identités de ce type n'en serait pas une, mais plusieurs. Si, comme le dit Quine ${ }^{4}$, il n'est d'entité sans identité, chaque entité ne dispose que d'une seule identité numérique. C'est donc certainement l'identité qualitative qu'ils évoquent, laquelle est effectivement susceptible d'être «plurielle». Un individu à l'âge de deux ans et le même individu à soixante ans sont numériquement identiques, mais ne le sont pas qualitativement. Ils sont la même entité, mais ne partagent pas certaines de leurs propriétés.

Le problème est qu'un individu ne peut avoir plusieurs identités qualitatives en même temps, car tout changement d'identité qualitative implique l'écoulement d'un laps de temps. L'identité qualitative est, en ce sens, une notion diachronique. Or, la théorie de l'identité plurielle soutient qu'un individu peut avoir plusieurs identités au même moment: chaque appartenance groupale confère à l'acteur social une identité; il évolue dans plusieurs groupes en même temps; donc il détient plusieurs identités simultanément. Mais ceci est formellement impossible, car l'identité qualitative est fixe à $\mathrm{t}_{0}$. Ce n'est qu'entre $\mathrm{t}_{0}$ et $\mathrm{t}_{1}$ qu'elle est susceptible de changer. La notion d' «identité plurielle» est donc une contradiction dans les termes.

On pourra objecter à cet argument que la conception plurielle de l'identité est métaphorique. Même si la notion d' «identité plurielle» est une contradiction logique, elle demeure utile pour penser la pluralité des insertions sociales dans les sociétés contemporaines. Une réponse à cette objection impliquerait de longs développements relatifs à la fonction du raisonnement analogique dans les sciences sociales, qui dépasseraient de beaucoup le cadre de cet article. Dans le cas présent, il nous suffit de dire que la métaphore est largement superflue. Comme nous aurons l'occasion de le montrer, la sociologie dispose de concepts qui permettent de l'éviter.

\section{L'identité construite}

La troisième conception de l'identité est l'identité construite. Celle-ci récuse les cinq caractéristiques énoncées précédemment, et défend l'idée que l'identité est une «construction sociale». Cette opinion participe d'une tendance de la sociologie contemporaine à considérer que tout est construit socialement. Sont construits non seulement les faits sociaux, mais l'ensemble de la réalité, y compris les objets naturels. Les théories «constructivistes» sont évidemment fort problé-

\footnotetext{
W. v. O. Quine, 1977, Le mot et la chose, Paris, Flammarion.
} 
matiques et ont fait l'objet de réfutations solides ${ }^{5}$. En matière de théorie de l'identité, elles soulèvent toutefois une difficulté particulière, sur laquelle il est intéressant de s'attarder.

L'idée qui se trouve au principe du «constructivisme» est qu'il n'existe pas de réalité indépendante de la perception qu'en ont les êtres humains. Transposée au cas qui nous occupe, elle implique qu'il n'existe pas d'identité en dehors de la perception que nous pouvons en avoir. Une telle position sous-tend notamment certains travaux de François de Singly, qui affirme par exemple que la famille contemporaine contribue à «construire les identités personnelles de chacun de ses membres», ou encore que «(...) la construction de l'identité personnelle dans les sociétés individualistes réclame (...) des soutiens venant d'autres individus disponibles.» ${ }^{6}$ En somme, il n'y a rien, dans la réalité, qui corresponde à des individus dotés d'une identité. Cette dernière n'existe pas à l'état naturel et est donc une «invention» de la société.

Ce point de vue pose un problème de taille. La thèse de la construction sociale soutient que la réalité est issue de nos états mentaux, en ce sens que sans eux, elle n'existerait pas. Les constructivistes en matière d'identité ajoutent que cette dernière est, elle aussi, une construction sociale, ce qui revient à dire que nos états mentaux sont eux-mêmes des constructions de la société. Mais il y a là une contradiction majeure. Car si nos états mentaux sont des constructions sociales, qui construit le monde qui nous entoure? Et qui construit nos états mentaux? Si l'individu n'existe pas, ou s'il n'est qu'une construction sociale, il s'ensuit que cette construction ne peut pas s'opérer, puisque c'est lui qui est censé en être le sujet (ou le «constructeur»). Il apparaît par conséquent qu'on ne peut être constructiviste à la fois en matière de réalité et d'identité personnelle. Si on l'est en ce qui concerne la première, il faut au moins préserver l'identité de la construction, car sans elle, aucune construction ne peut avoir lieu. Mais dans la mesure où le constructivisme affirme que tout est construit socialement, on ne voit pas en vertu de quoi il pourrait tenir l'identité personnelle hors de portée de cette construction.

\section{LE PROBLÈME DE L'IDENTITÉ PERSONNELLE}

Aucune des conceptions de l'identité présentées ci-dessus ne semble satisfaisante. Chacune manque soit de cohérence interne, soit de congruence avec la réalité. Le concept d'identité, il est vrai, est particulièrement problématique. La sociologie n'est d'ailleurs pas la seule à être confrontée à la difficulté d'en fournir une définition rigoureuse. Le débat concernant l'identité personnelle est l'un des plus fréquentés de la philosophie contemporaine. Un détour par les théories élaborées dans ce domaine contribuera à éclaircir les enjeux relatifs à l'utilisation de ce concept.

\footnotetext{
Ian Hacking, 2000, The Social Construction of What?, Cambridge, Harvard University Press.

6 François De Singly, «La fabrique familiale de soi», in Jean-Claude Ruano-Borbalan (éd.), op. cit., p. 173 .
} 


\section{Les origines}

Le premier philosophe moderne à avoir posé le problème de l'identité personnelle est John Locke. La définition qu'il en propose est simple: «(Person stands for) a thinking intelligent being, that has reason and reflection, and can consider itself as itself, the same thinking thing, in different times and places; which it does only by that consciousness which is inseparable from thinking (...).» ${ }^{7}$ Pour Locke, la conscience est le fondement de l'identité personnelle. Tout individu dispose d'un accès privilégié à ses propres états mentaux, en ce sens qu'il est le seul à pouvoir les atteindre sur le mode du «je». Si d'autres personnes peuvent accéder à ses pensées, nul, hormis lui-même, ne peut les connaître «à la première personne». Il suffit par conséquent qu'il soit conscient d'être une personne pour être cette personne. Dans la mesure où la conscience porte sur des faits aussi bien présents que passés, le critère de l'identité doit être élargi à la mémoire.

La thèse lockéenne de la continuité mémorielle contredit deux doctrines. La première est le substantialisme, selon lequel l'identité est une entité immatérielle irréductible aux états psychologiques. Cette position est notamment celle Descartes, qui défend l'idée qu'il y a quelque chose de plus dans l'identité personnelle que le seul fait d'être conscient. Ce fait supplémentaire, c'est l'âme, qu'il est impossible de réduire à une succession d'états mentaux, et qui constitue le «noyau» de la personne. Contrairement à celle de Descartes, la thèse de Locke est réductionniste. Pour le philosophe britannique, il n'y a aucun fait primitif à la base de l'identité.

La seconde théorie à laquelle s'oppose Locke est le réductionnisme physique. Selon ce dernier, le critère de l'identité personnelle n'est pas la continuité mémorielle, mais la continuité corporelle. Le réductionnisme physique est bien un réductionnisme, en ce sens qu'il considère l'identité personnelle comme une entité décomposable. Mais contrairement à Locke, ses partisans soutiennent que c'est dans l'organisation physique des individus qu'il faut chercher le principe de leur identité, et non dans leur organisation psychologique.

Pour plausible qu'elle paraisse, la théorie de l'identité de Locke n'en a pas moins fait l'objet de plusieurs objections. La première est due à Joseph Butler qui, dans The Analogy of Religion, avance l'argument suivant: «And one should really think it self-evident, that consciousness of personal identity presupposes, and cannot therefore constitute, personal identity any more than knowledge, in any other case, can constitute truth, which it presupposes.» ${ }^{8}$ Pour Butler, l'idée que la mémoire fonde l'identité personnelle est fausse. Plus exactement, elle est circulaire, car la conscience présuppose l'identité. Il faut que la personne existe pour qu'elle puisse avoir conscience d'elle-même. Comment, dans ces conditions, la mémoire pourrait-elle constituer l'identité personnelle? De même que la connaissance implique la vérité (au sens où il n'est de connaissance que vraie), de même, la mémoire implique l'identité.

John Locke, 1690, Essay Concerning Human Understanding, chapitre 27, reproduit dans John Perry (éd.), 1976, Personal Identity, Berkeley, University of California Press, p. 39.

8 Joseph Butler, 1736, The Analogy of Religion, première dissertation, cité par David Wiggins, «Locke, Butler and the Stream of Consciousness : and Men as a Natural Kind », in Amelie Oksenberg Rorty (éd.), 1976, The Identities of Persons, Berkeley, University of California Press, p. 141. 
Une seconde objection a été adressée à Locke par Thomas Reid. Celle-ci repose sur un thought experiment fameux, connu sous le nom d'expérience du «brave officier ${ }^{9}$. Un brave officier a été fouetté dans son enfance parce qu'il avait volé des fruits dans un verger. Ce même officier a remporté, lors de sa première campagne militaire, une bataille prestigieuse. A un âge plus avancé, il a été élevé au grade de général. Imaginons que lorsqu'il remporta la bataille, l'officier se souvenait avoir été fouetté dans son enfance. Au moment où il devint général, il avait le souvenir d'avoir gagné la bataille, mais n'avait plus conscience d'avoir été fouetté.

Selon Reid, ce cas trivial remet en cause la base même de la théorie de Locke. Si le critère de l'identité personnelle est la continuité mémorielle, c'est-à-dire le fait pour un individu de se souvenir de ses actions passées, il s'ensuit que le général et l'enfant fouetté ne sont pas la même personne. Plus précisément, l'officier qui remporta la bataille prestigieuse et l'enfant puni sont bel et bien la même personne, puisque l'individu final (l'officier) se souvient de l'individu initial (l'enfant). De même, le général et l'officier sont la même personne, puisque le général a encore conscience d'avoir remporté la bataille prestigieuse. Mais le général et l'enfant ne peuvent être considérés comme identiques, puisque le premier ne se souvient pas du second. La théorie lockéenne viole en fait une propriété importante de la relation d'identité: la transitivité, selon laquelle si $\mathrm{x}=\mathrm{y}$ et $\mathrm{y}=\mathrm{z}$, alors, $\mathrm{x}=\mathrm{z}$. Si l'enfant est identique à l'officier, et si l'officier est identique au général, alors l'enfant devrait être identique au général. Mais ceci n'est pas le cas, car la continuité mémorielle que retient Locke comme critère de l'identité est une relation intransitive.

\section{Positions contemporaines}

La thèse de la continuité psychologique de Locke s'est donc vue opposer des objections puissantes de la part de Butler et Reid. Les arguments proposés par ces derniers ont conduit bon nombre de philosophes à renoncer au critère de la mémoire, et à chercher les conditions d'identité de la personne du côté de ses propriétés matérielles. Ces recherches ont donné lieu à plusieurs théories, qui ont en commun l'idée que demeurer la même personne à travers le temps, c'est essentiellement demeurer la même entité physique.

La première de ces doctrines est le réductionnisme corporel, selon lequel c'est la continuité corporelle qui est le critère de l'identité personnelle. Autrement dit, une personne $\mathrm{A}$ à $\mathrm{t}_{0}$ est identique à une personne $\mathrm{B}$ à $\mathrm{t}_{1}$ si et seulement si $\mathrm{A}$ et $\mathrm{B}$ ont le même corps. Cette idée est congruente avec le sens commun. La plupart de nos assignations ordinaires d'identité pourraient aisément s'expliquer par ce critère. Cependant, elle tombe sous le coup d'objections évidentes. Qu'advient-il de l'identité d'une personne lorsqu'elle meurt? Si l'on s'en tient au réductionnisme corporel, une personne qui meurt reste la même personne, puisque au moment de sa mort, son corps ne disparaît pas (du moins pas immédiatement). Mais ceci n'a évidemment aucun sens.

9 Thomas Reid, 1785, Essays on the Intellectual Powers of Man, premier essai, reproduit partiellement dans Stéphane Ferret (éd.), 1998, L'identité, Paris, Flammarion. 
Le second type de réductionnisme physique est le réductionnisme cérébral, qui affirme que la condition de persistance d'un individu dans le temps est la permanence de son cerveau. $\mathrm{Si}$, pour une raison ou une autre, ce dernier venait à être altéré, l'individu ne serait plus identique à lui-même.

Il existe de nombreuses raisons de rejeter cette conception. Imaginons que dans quelques années, la science soit capable d'inventer des cerveaux artificiels ${ }^{10}$. Supposons qu'une tumeur maligne se soit installée dans mon cerveau. Mon médecin décide de mettre à profit cette nouvelle technologie, et de substituer progressivement aux cellules cancéreuses des cellules de silicone. Mon cerveau est ainsi entièrement remplacé par un cerveau artificiel. Pendant l'opération, mon activité mentale n'a cependant connu aucun changement. Je continue à avoir les mêmes croyances, les mêmes désirs et les mêmes projets qu'auparavant.

Deux conclusions peuvent être tirées de cette expérience. On peut constater, à juste titre, que mon cerveau final n'est pas identique à mon cerveau initial. Si l'on défend une position «cérébraliste» en matière d'identité personnelle, il faut en conclure que la personne post-opératoire n'est pas la même personne qu'avant. Mais cette conclusion paraît douteuse, car nous avons admis, par hypothèse, que mon activité cérébrale ne s'est pas interrompue au moment de la substitution des cellules. Compte tenu de ce fait, il semble que je puisse survivre sans mon cerveau originel. Le critère de la continuité cérébrale n'est donc pas suffisant pour fonder l'identité personnelle.

\section{Le réductionnisme de Derek Parfit}

A ce stade, les critères psychologiques et physiques de l'identité semblent également compromis. Plusieurs philosophes contemporains ont tâché d'en proposer des formulations qui les préservent des arguments présentés ci-dessus. La plus intéressante et la plus convaincante de ces formulations est celle de Derek Parfit, qui concerne le critère psychologique.

Conscient des difficultés soulevées par la position de Locke, Parfit entreprend de la modifier. En premier lieu, il affirme que le critère de la mémoire n'est pas le seul à même de conférer à une personne ses conditions de persistance dans le temps. Il existe d'autres formes de continuité psychologique capable de la préserver. Ainsi du rapport entre les intentions et les actions: si j'accomplis une action au temps $t_{1}$, c'est parce qu'au temps $t_{0}$, j'ai eu l'intention d'agir de la sorte. Toute action présuppose l'existence préalable d'une intention de la part de l'individu. Ceci confère à ce dernier une inscription dans le temps, dans la mesure où une certaine période s'écoule nécessairement entre la formation de l'intention et sa réalisation.

En second lieu, Parfit soutient que l'objection de circularité émise par Butler à l'encontre de Locke peut être contournée. Supposons que j'ai le souvenir de m'être cogné la tête contre un mur à l'âge de deux ans. Je peux avoir ce souvenir parce que je m'en souviens effectivement, tout comme je me souviens m'être cogné la tête contre une porte la semaine passée. Mais je peux aussi ne pas avoir de cet événement un souvenir direct, et le posséder parce que ma mère me l'a

10 Brian Garrett, 1998, Personal Identity and Self-Consciousness, Londres et New York, Routledge, p. 49. 
raconté. Dans ce dernier cas, j'ai le souvenir de m'être cogné la tête parce que ce dernier a été causé dans mon esprit par l'histoire que m'a racontée ma mère, et non par l'événement lui-même.

Peut-on considérer ce souvenir comme authentique? Doit-on au contraire admettre qu'il est artificiel, puisqu'il m'a été rapporté par autrui? Selon Parfit, il est possible de lui reconnaître le statut de souvenir, c'est-à-dire, plus exactement, de quasi-souvenir. Un quasi-souvenir a les trois conditions de possibilité suivantes $^{11}$ :

1. l'individu qui le porte a l'impression d'avoir vécu l'événement qui fait l'objet du quasi-souvenir;

2. quelqu'un a effectivement vécu l'événement en question;

3. l'impression de l'individu découle d'une cause appropriée.

Les quasi-souvenirs existent-ils réellement? Ce qui est certain, c'est que des cas de figure comme celui où je me «quasi-souviens » m'être cogné la tête contre un mur à l'âge de deux ans sont fréquents. L'objectif que vise Parfit avec ce concept est manifeste. Il s'agit de prendre le contre-pied de l'objection de Butler selon laquelle toute mémoire présuppose l'existence de l'individu qui la détient. $\mathrm{Si}$ les quasi-souvenirs existent, il devient possible de dissocier un souvenir du sujet qui l'éprouve. En d'autres termes, une description du souvenir en des termes strictement impersonnels se révèle concevable. Dans ces conditions, le critère de continuité mémorielle ne présuppose plus l'existence de la personne, et n'est donc plus circulaire.

Grâce au concept de quasi-souvenir, Parfit semble être à même d'esquiver la critique de Butler. Mais qu'en est-il de celle de Thomas Reid? Ce dernier, on s'en souvient, affirmait qu'il y a une contradiction entre le caractère intransitif de la continuité mémorielle, et le fait que l'identité est une relation transitive. Pour répondre à cette objection, Parfit propose de distinguer la connectivité et la continuité mémorielle ${ }^{12}$. Il y a connectivité mémorielle entre $\mathrm{X}$ et Y si Y peut se souvenir directement d'un événement vécu par X. Ce concept désigne en fait ce que Locke appelait la continuité de mémoire. Le problème, nous l'avons vu, est que la connectivité est une relation intransitive. Parfit, dès lors, lui substitue la relation de continuité, qui, dans son acception, consiste en l'existence entre deux individus de connexions mémorielles abondantes. La continuité ainsi définie peut être, dans certains cas, une relation transitive. Si X est fortement connecté à Y, et Y est fortement connecté à $\mathrm{Z}$, alors il peut exister une continuité psychologique entre $\mathrm{X}$ et $\mathrm{Z}$.

Mais qu'entendre exactement par connectivité abondante? C'est tout le problème. La fixation d'un seuil unique est impossible. Le seul élément de réponse que l'on peut avancer est qu'il doit exister entre un individu $\mathrm{X}$ et un individu Y suffisamment de connexions pour qu'ils soient le même individu. Si, par exemple, Y ne se souvient que d'un seul événement survenu à $\mathrm{X}$, la connectivité est clairement insuffisante. Dans ce cas, Y n'est pas la même personne que X. Si, en

11 Derek Parfit, 1984, Reasons and Persons, Cambridge, Cambridge University Press, p. 220.
12 Ibid., p. 206. 
revanche, Y se souvient d'un nombre important d'événements survenus à X, alors ces deux personnes peuvent être considérées comme identiques.

Pour Parfit, l'identité personnelle est tributaire des circonstances dans lesquelles elle est assignée. Il n'existe pas de seuil immuable de continuité psychologique au-delà duquel une personne est nécessairement la même personne. L'identité personnelle est donc une question de degré. Si une personne consiste en la continuité de certains de ses états mentaux, et si cette continuité peut souffrir de degrés, alors l'identité personnelle est, elle aussi, une relation graduelle. Je suis aujourd'hui plus ou moins la même personne qu'hier. De même, je serai plus ou moins la même personne demain que celle que je suis actuellement.

Le problème de l'identité personnelle est en fait similaire à ce que les philosophes appellent les arguments sorites. Considérons le concept de «tas ». Dix millions de grains de sable forment de toute évidence un tas de sable. Dix millions moins un grains de sable également, de même que dix millions moins deux, trois, quatre... n - 1 grains de sable. Donc, un grain de sable forme un tas de sable.

Cette conclusion est clairement paradoxale, mais on voit mal comment il est possible de l'éviter. Ce qui est certain, c'est que ni l'objet «sable», ni l'objet «tas» ne peuvent nous aider à le résoudre. Autrement dit, la réponse à ce problème n'est en aucun cas une réponse factuelle. C'est une réponse stipulée. Comme le dit Parfit, "We know that the concept of a heap is vague, with vague borderlines. And when the Sorites Argument is applied to heaps, we are happy to solve the problem with a stipulation: an arbitrary decision about how to use the word "heap".» ${ }^{13} \mathrm{Il}$ n'est d'autre manière de déterminer ce que désigne le concept de «tas» que de stipuler ce qu'il recouvre. De même, il n'est d'autre moyen de savoir ce que dénote le concept de personne que de stipuler ce qu'il dénote. Ceci ne vaut évidemment pas dans tous les cas. Il existe parfois des connectivités si fortes que la question de savoir si une personne est identique à elle-même va de soi. Mais dans d'autres cas, l'acte que constitue la stipulation est nécessaire pour conférer son identité à une personne.

\section{L'INFRA-INDIVIDUALISME MÉTHODOLOGIQUE}

Le problème de l'identité personnelle, on le voit, est complexe. Il l'est à ce point que la théorie philosophique la plus aboutie le concernant propose l'abandon presque complet de ce concept. Les thèses de Derek Parfit ont rencontré un certain écho dans les sciences sociales. L'une des doctrines où cette influence s'exprime le plus clairement est la théorie du «soi multiple», élaborée par des auteurs comme Jon Elster, Georges Ainslie ou Amos Tversky ${ }^{14}$. Nous commencerons par présenter cette théorie, afin de tester la pertinence d'un renoncement au concept d'identité personnelle, puis montrerons qu'un tel abandon pose plus de problèmes qu'il n'en résout.

3 Ibid., p. 232.

14 Le texte le plus représentatif de ce courant, The Multiple Self, est paru deux ans après Reasons and Persons, l'ouvrage principal de Parfit. Plusieurs des auteurs qui y ont contribué font explicitement référence à ce dernier. 


\section{La théorie du soi multiple}

L'idée qui se trouve au fondement de la théorie du soi multiple est que toute personne est un agrégat d'entités infra-individuelles relativement autonomes. Cette doctrine affirme qu'il n'existe pas à proprement parler d'individus, mais seulement des soi «parallèles » emboîtés les uns sur les autres. La conséquence méthodologique de cette idée est qu'il convient de situer «en dessous » de l'individu le niveau général de l'explication sociologique. C'est pourquoi on peut parler à son propos d'infra-individualisme méthodologique.

Une première version de la théorie du soi multiple est celle des soi «désintégrés ». Selon elle, l'esprit fonctionne sur le modèle de la firme. Toute firme est constituée d'un certain nombre d'unités de production. Chaque firme comprend également une direction, chargée de coordonner les activités des différentes unités. Or, il arrive parfois que ces dernières échappent au contrôle de la première. Cela survient notamment lorsque les unités refusent de partager certains renseignements dont elles disposent. Si une unité détient des informations grâce auxquelles elle sait pouvoir augmenter sa productivité, elle peut être tentée de ne pas les transmettre à sa hiérarchie, au détriment de la productivité générale de la firme.

L'esprit rencontre parfois des problèmes similaires. Imaginons un enfant qui croit au père Noël et qui, en même temps, remet à ses parents une liste des cadeaux qu'il souhaite recevoir à l'occasion des fêtes de fin d'année. Sa croyance et cette action sont clairement antinomiques. Toutefois, les cas où une personne maintient dans son esprit des états mentaux mutuellement exclusifs sont fréquents. Ils finissent d'ailleurs souvent par se rencontrer. L'individu, prenant conscience de la contradiction, abandonne l'une de ses croyances. Mais il arrive aussi que cette «compartimentalisation» mentale se révèle plus durable, et conduise à l'émergence de plusieurs « unités cérébrales » indépendantes ${ }^{15}$.

Une seconde variante de la théorie du soi multiple est celle des soi «faustiens ». Cet adjectif désigne l'éventualité qu'un individu soit partagé entre plusieurs désirs. Son esprit ne détient pas, comme dans le cas précédent, des croyances opposées, mais des volontés contradictoires, dont les conditions objectives de satisfaction sont impossibles à concilier. Les cas de désirs antagonistes sont courants, et il serait abusif de dire qu'ils conduisent tous à des «clivages » du soi. Les problèmes surviennent lorsqu'un individu détient plusieurs désirs incompatibles en même temps. Par exemple, je peux à la fois désirer être en bonne santé et continuer à fumer des cigarettes, ou vouloir être une personne moralement irréprochable et céder régulièrement à des vices que je réprouve. Dans de tels cas, mon esprit peut subir une division, qui s'exprime la plupart du temps par l'accomplissement d'actions contradictoires. Si je persiste à fumer et que, parallèlement, je prêche l'abstinence en matière de cigarettes, on peut soutenir que ces deux comportements relèvent de soi autonomes.

La troisième forme que revêt l'hypothèse du soi multiple est celle des soi successifs. Imaginons un jeune idéaliste qui craint de devenir un vieillard cynique. Il considère que s'il renonce à son idéalisme, il sera devenu une autre personne. Il reconnaît toutefois que la probabilité que cela arrive est très grande. Il demande

15 Jon Elster, «Introduction», in Jon Elster (éd.), 1986, The Multiple Self, Cambridge, Cambridge University Press, p. 5. 
par conséquent à l'un de ses amis de faire ce qui est en son pouvoir pour le ramener, le cas échéant, à son soi précédent. Il lui promet une importante somme d'argent à cet effet. Selon certains théoriciens du soi multiple, on peut affirmer que le jeune idéaliste et l'hypothétique vieillard sont deux soi à part entière. La preuve en est que le soi initial - le jeune - considère qu'en devenant cynique, il se transformerait en une autre personne.

Quel crédit accorder à l'hypothèse du soi multiple? Elle pose de nombreux problèmes. Tout d'abord, soutenir que certains comportements témoignent de contradictions cognitives chez l'individu est une chose, en déduire l'existence de soi durablement autonomes dans son esprit en est une autre. Rares sont les cas qui impliquent un constat de cet ordre. Par conséquent, comme le reconnaît Jon Elster, «(...) we ought not to take the notion of "several selves" literally. In general, we are dealing with exactly one person - neither more or less.» ${ }^{16}$ Le second problème est que dans bien des cas où cette hypothèse est invoquée, il semble possible de lui en substituer une autre, à la fois moins lourde et plus cohérente. On évoquera, à titre d'exemple, l'analyse du paradoxe du vote proposée par les psychologues Amos Tversky et Georges Quattrone ${ }^{17}$.

\section{Le paradoxe du vote}

Tversky et Quattrone ont proposé une solution intéressante au fameux paradoxe du vote ${ }^{18}$. Elle consiste à distinguer entre deux types de causalité: la causalité instrumentale et la causalité diagnostic. Considérons l'exemple suivant. Les calvinistes croient en la prédestination, qui a deux conséquences importantes pour eux. D'une part, elle partage les êtres humains en deux catégories: les élus et les non élus. D'autre part, elle a pour résultat que les premiers vivront une existence conforme à la morale, et les seconds une vie impie. Or, les calvinistes ne savent qui est élu et qui ne l'est pas. De surcroît, le fait d'être élu n'est pas de leur ressort, puisqu'il est déterminé par Dieu. Malgré cela, ils décident de vivre une existence moralement irréprochable.

La décision des calvinistes d'agir en conformité avec la morale est parfaitement irrationnelle. Leur comportement n'a, selon leur propre doctrine, aucun effet sur leur élection. S'ils décident de vivre dans la piété, c'est, selon Tversky et Quattrone, parce qu'ils confondent la causalité instrumentale et la causalité diagnostic. La première désigne la causalité ordinaire. En l'occurrence, la prédestination est une cause instrumentale de l'élection, qui est elle-même une cause instrumentale de la vie morale. La seconde est une causalité symptomatique: le fait de se comporter moralement est un signe de l'élection, même si elle n'en est pas une cause matérielle.

Le problème est que les deux propositions auxquelles adhèrent les calvinistes sont contradictoires. On ne peut à la fois croire que l'élection est prédéterminée, et que l'acte de vivre moralement est motif d'élection. Pourtant, les calvinistes

16 Ibid., p. 30.

17 Amos Tversky et Georges Quattrone, «Self-deception and the voter's illusion», in Jon Elster (éd.), op. cit., pp. 35-58.

18 Les individus sont rationnels. Ils ne devraient donc pas aller voter, car l'impact d'un bulletin sur le scrutin est nul. Or, on observe que les individus vont voter. Ils sont donc irrationnels. 
parviennent bel et bien à maintenir dans leur esprit ces deux croyances mutuellement exclusives. Selon Tversky et Quattrone, pour expliquer ce fait surprenant, on peut faire appel aux phénomènes de «compartimentalisation» mentale évoqués ci-dessus. Ils examinent principalement l'un de ces mécanismes: la «duperie de soi » (self-deception), qui désigne les cas où un individu détient deux croyances contradictoires dont l'une est motivée par un désir.

Le paradoxe du vote s'explique aisément à partir de ce modèle. Le vote individuel a une conséquence infime sur le résultat du scrutin. La personne n'en escompte aucun bénéfice, tout en supportant son coût. Si elle se rend à l'urne, c'est parce qu'elle considère son vote comme un diagnostic du fait que d'autres individus, notamment des individus qui ont la même opinion qu'elle, iront voter. Elle perçoit son acte comme symptomatique de l'attitude des autres électeurs: si elle ne va pas voter, les autres n'y iront pas non plus, mais si elle se rend à l'urne, les autres s'y rendront également.

Cette thèse est indéniablement séduisante. Toutefois, il semble possible de lui substituer une explication à la fois plus simple et moins ad hoc: 1'analyse en terme de rationalité axiologique. Selon Raymond Boudon ${ }^{19}$, une résolution du paradoxe pourrait prendre pour point de départ le concept weberien de Wertrationalität. Dans l'acception de Boudon, la rationalité axiologique est la version prescriptive de la rationalité cognitive, qui explique les croyances et les comportements par les «bonnes raisons » des acteurs sociaux ${ }^{20}$.

Comment la rationalité axiologique résout-elle le paradoxe du vote? Pour Boudon, les individus ont d'excellentes raisons de croire que la démocratie est un bon régime politique. C'est un bon régime, en particulier, parce qu'il permet à la volonté populaire de s'exprimer. Or, pour que cette volonté apparaisse, il faut que les citoyens se manifestent lors des scrutins. Leur participation aux élections relève donc d'un impératif moral. Comme le dit le sociologue: «(..) en appliquant le principe "il faut voter" (sauf si j" ai des raisons fortes de ne pas l'appliquer), je fais ce qui est en mon pouvoir pour que la consultation se déroule normalement. C'est parce que le principe "il faut voter" est fondé sur des raisons solides que j' ai le sentiment que je dois m'y conformer.» ${ }^{21}$

Cette explication semble meilleure que celle de Tversky et Quattrone. Surtout, elle évite de s'en remettre à une hypothèse aussi coûteuse que celle du soi multiple. Or, comme nous l'avons dit, bon nombre des cas invoqués par ses partisans peuvent faire l'objet d'une reformulation de ce type. Si tel est le cas, il semble peu probable que l'infra-individualisme qu'ils préconisent soit une option théorique avantageuse.

Il est une raison épistémologique plus profonde de maintenir l'individu comme catégorie sociologique de base. On ne peut considérer comme rationnels que des individus à part entière. Les entités infra-individuelles qu'invoque la théorie du soi multiple fonctionnent sur un mode causal, et non rationnel. Or, si l'on considère que l'analyse par les raisons est le type d'explication privilégié des

\footnotetext{
19 Raymond Boudon, 1997, «Le "paradoxe du vote" et la théorie de la rationalité », in Revue française de sociologie, vol. 38.

20 Raymond Boudon, 1986, L’idéologie, ou l'origine des idées reçues, Paris, Fayard.

21 Raymond Boudon, «Le "paradoxe du vote" et la théorie de la rationalité », op. cit., p. 223.
} 
sciences sociales, et si l'on reconnaît que seuls des individus peuvent être rationnels, alors, il faut conserver ces derniers comme catégorie d'analyse.

Des entités infra-individuelles ne peuvent-elles jamais être considérées comme rationnelles? Il arrive que les théoriciens du soi multiple parlent des «stratégies » mises en œuvre par l'une ou l'autre des parties opposées d'un esprit. Ils évoquent parfois la «prise de contrôle» de l'une de ces parties par l'autre. En ce sens, on pourrait admettre que ces entités possèdent une forme de «rationalité », tout comme, dans d'autres contextes, on peut soutenir que les gènes font preuve d' «égoïsme», ou que certains animaux oligocellulaires sont doués d'une "posture intentionnelle ${ }^{22}$. Mais de deux choses l'une: ou bien ces usages de la notion de rationalité sont métaphoriques, ou alors le concept de rationalité y est utilisé en un sens minimaliste. Ce qui semble certain, c'est que des formes complexes de rationalité comme les rationalités cognitive et axiologique ne peuvent être assignées qu'à des personnes. Un «infra-individu» ne peut en aucun cas avoir de «bonnes» ou de «mauvaises» raisons d'effectuer telle action, ou d'adopter telle croyance. L'application du concept de rationalité implique donc le rejet de l'«infra-individualisme», et la prise en charge d'une ontologie sociale au moins individualiste.

\section{IDENTITÉ PERSONNELLE ET INTERACTIONS SOCIALES}

Puisque l'individu doit être maintenu comme concept sociologique fondamental, il semble légitime de conserver également la notion d'identité personnelle. En effet, on ne voit pas comment on pourrait faire usage de la notion d'individu sans la lier, d'une façon ou d'une autre, à celle d'identité. Un individu dispose toujours d'une certaine forme d'identité, quel que soit le sens précis que l'on confère à ce terme. Or, le problème est que la critique de l'identité personnelle développée par Derek Parfit est fort convaincante. Comment concilier la position du philosophe avec l'idée que l'individu et son identité doivent être préservés?

Dans La logique du social, Raymond Boudon insiste sur le caractère réciproque des liens existant entre un individu et l'environnement dans lequel il évolue $^{23}$. Par l'entremise des concepts de processus «reproductif», «cumulatif» et «transformatif», il explique la façon dont les actions et les croyances des acteurs façonnent les structures d'interaction, et la manière dont, en retour, ces dernières influent sur leurs actions et croyances. Si je suis un chef d'entreprise qui dirige une «start-up» dans les années 1990, mes croyances ne seront pas les mêmes que si je dirige une entreprise d'Etat dans les années 1960. Dans le premier cas, je serai par exemple conduit à adhérer à des méthodes managériales «libertaires », dans le second à des méthodes plutôt «paternalistes». A l'inverse, il est clair que mes croyances et comportements influeront sur l'entreprise que je dirige.

Tout système d'interaction confère aux individus certaines propriétés. Plus précisément, le fait que l'individu détienne ces propriétés a pour condition néces-

22 Richard Dawkins, 1978, Le gène égoïste, Paris, Mengès, et Daniel Dennett, 1990, La stratégie de l'interprète, Paris, Gallimard.

23 Raymond Boudon, 1979, La logique du social, Paris, Hachette. 
saire son insertion dans un système d'interaction. Si je suis chef d'entreprise, j'ai des propriétés comme celles de gagner beaucoup d'argent, d'avoir du pouvoir sur mes subordonnés, et d'être en position d'infléchir la stratégie de mon groupe. Si, de surcroît, je suis chef d'entreprise dans les années 1990, j'ai certaines qualités que mon homologue des années 1960 n'avait pas, ou ne jouis pas de certains attributs dont lui disposait.

On peut tirer trois conclusions de ces brèves considérations. Tout d'abord, le fait pour un individu de se trouver dans un système d'interaction lui confère certaines propriétés. Celles-ci ne sont pas intrinsèques, puisqu'une personne isolée ne pourrait les détenir. Ce sont des propriétés relationnelles, c'est-à-dire, plus précisément, des propriétés sociales, en ce sens qu'elles sont la conséquence de l'insertion de l'individu dans un système d'interaction comprenant d'autres individus.

La seconde conclusion est que les systèmes d'interaction se modifient avec le temps. Les qualités d'un chef d'entreprise dans les années 1960 ne sont pas les mêmes que celles d'un entrepreneur des années 1990. Les changements interviennent, d'une part, par l'entremise des croyances et des comportements des acteurs eux-mêmes. D'autre part, des modifications peuvent survenir du fait d'une transformation de l'environnement dans lequel se trouve le système considéré.

La troisième conclusion est que malgré les changements qui s'opèrent en leur sein, les systèmes sociaux sont doués d'une certaine inertie. D'un point de vue général, les modifications qu'ils subissent sont lentes, ce qui fait d'eux des entités relativement stables. Etre chef d'entreprise dans les années 1960 et l'être dans les années 1990 n'implique pas la reproduction des mêmes comportements. Certains attributs de cette fonction ont changé avec le temps. La plupart cependant, constitutifs de la fonction de chef d'entreprise, sont demeurés similaires.

La problématique de l'identité personnelle, on s'en souvient, concerne les conditions de persistance d'un individu dans le temps : comment savoir si une personne à $\mathrm{t}_{1}$ est la même personne qu'à $\mathrm{t}_{0}$ ? $\mathrm{Il}$ y a fort à parier que l'insertion des individus dans des groupes sociaux contribue largement à leur persistance dans le temps. Pourquoi? Parce que ces groupes sont stables, de même que les propriétés qu'ils confèrent à leurs membres. Or, affirmer que les propriétés sociales sont stables revient simplement à dire qu'elles sont identiques entre $t_{0}$ et $t_{1}$. Dans la mesure où ces propriétés ont pour objet les individus, cette identité se transmet nécessairement à eux. Par conséquent, si du point de vue philosophique on peut admettre que l'identité d'une personne est indéterminée, il faut soutenir qu'elle ne l'est nullement du point de vue sociologique. Il semble dès lors possible de circonscrire un troisième critère de l'identité personnelle, le critère social, qui constitue une alternative aux critères psychologique et physique traditionnellement retenus pour définir l'identité personnelle.

Nous avons mis en question ci-dessus certains usages contemporains du concept d'identité. Le critère social ne tombe-t-il pas sous le coup de ces critiques? Il semble que non. En particulier, son application aux individus n'empêche nullement de considérer ces derniers comme rationnels. Pour démontrer ce point, on peut faire appel à une notion classique de la science sociale: celle de rôles, qui se définit comme «(...) systèmes de contraintes normatives auxquelles 
sont censés se plier les acteurs qui les détiennent, et de droits corrélatifs à ces contraintes.» ${ }^{24}$

Le rôle est un «système de contraintes » dont hérite chaque individu appartenant à un groupe. Le fait d'être directeur d'entreprise en est un exemple typique. Tout directeur a comme contrainte relative à son rôle celle de faire croître son entreprise. Un droit corrélatif à ce devoir est celui de gagner plus d'argent qu'un employé dont l'impact sur la firme est moindre. Ces droits et devoirs renvoient, d'une part, à des faits, c'est-à-dire à la façon dont se passent les choses dans la réalité. D'autre part, ce sont des normes idéales auxquelles les acteurs sociaux se plient de façon plus ou moins rigoureuse.

Dès lors que l'on admet une certaine latitude dans l'exécution des rôles sociaux, on confère à la rationalité des acteurs un espace d'expression. Affirmer que le chef d'entreprise hérite d'un système de contraintes n'équivaut nullement à soutenir que ses comportements sont «archétypaux». Cela revient simplement à défendre l'idée que la rationalité du chef d'entreprise s'exerce dans le cadre de normes et de valeurs avec lesquelles il est obligé de composer. Toute rationalité, qu'elle soit instrumentale, cognitive, ou axiologique doit s'accommoder de données objectives sur lesquelles la personne n'a pas prise.

Un individu est susceptible de se voir assigner de nombreux rôles. Il est possible de «jouer» simultanément les rôles de dirigeant d'entreprise, de père de famille, de partenaire de golf et d'adhérent à un parti politique. Pour désigner ce phénomène, Robert Merton a proposé la notion de role-sets, définie comme l'ensemble des rôles sociaux qui incombent à un individu donné ${ }^{25}$. Chaque rôle a évidemment une importance variable pour la personne qui le porte, alors que les exigences qu'il lui impose peuvent, dans certains cas, entrer en contradiction.

Nous avons déjà envisagé la question de la multiplicité des rôles sociaux lorsque nous avons soumis à critique le concept d' «identité plurielle». La théorie de l'identité plurielle, on s'en souvient, affirmait que puisque les individus sont insérés dans de nombreux groupes sociaux, ils sont des êtres «pluri-identitaires». Le problème, avions-nous dit, est que l'identité ne peut en aucun cas être multiple. Une entité qui détiendrait plusieurs identités n'en serait pas une, mais plusieurs. Cette contradiction peut être dépassée à l'aide du concept de rôle. Il n'est en effet pas antinomique de soutenir qu'un seul individu a de multiples rôles sociaux. Chacun de ces rôles lui est conféré par l'un des collectifs auxquels il appartient, ce qui le conduit à endosser successivement des comportements d'inspiration sociale différente. Lorsque la multiplicité des rôles est contradictoire, l'acteur social opère un arbitrage (conscient ou inconscient) en faveur de l'un ou l'autre des rôles auxquels il doit se plier.

D’un point de vue général, les rôles peuvent être considérés comme des mécanismes de stipulation de l'identité personnelle. Les propriétés sociales dont ils sont porteurs permettent de déterminer les cas où il convient d'assigner une identité à une personne, et ceux où cette attribution n'est pas pertinente. Puisque les

24 Article «Rôle», in Raymond Boudon et François Bourricaud (éds.), 1982, Dictionnaire critique de la sociologie, Paris, PUF.

25 Robert Merton, 1976, Sociological ambivalence and other essays, New York et Londres, The Free Press. 
rôles sociaux ont pour caractéristique de conférer des propriétés durables aux individus, ils contribuent à «stabiliser», pour un certain temps au moins, leur identité.

Imaginons une personne ayant la propriété de diriger une entreprise. Cette personne a conclu, par le passé, des contrats commerciaux, mais ne se souvient ni du détail de ces contrats, ni de l'ordre dans lequel elle les a signés. A vrai dire, elle a oublié jusqu'à l'existence de certains d'entre eux. Si l'on s'en tient à la théorie de Derek Parfit, le directeur actuel de cette firme n'est pas la même personne que celle qui a signé les contrats oubliés. La raison en est que l'identité personnelle présuppose la continuité mémorielle, et que cette dernière est inexistante dans ce cas. De plus, la question de savoir si le directeur est la même personne que celle qui a signé les contrats dont les détails lui échappent est indéterminée. Car il est impossible en l'occurrence de mesurer précisément le degré de continuité mémorielle existant entre la personne actuelle et la personne passée, et donc impossible d'établir entre elles une relation d'identité.

Pour philosophiquement acceptable qu'il soit, ce raisonnement est sociologiquement absurde. Pourquoi? Parce que tout, dans l'environnement social du directeur, contribue à lui remémorer les contrats. Sa qualité de directeur aura pour conséquence, par exemple, que ses employés viendront régulièrement l'interroger sur le contenu des contrats en question. De ce fait, ces derniers seront constamment rappelés à sa conscience. Un rôle social comme celui de «directeur» peut ainsi lever l'indétermination de l'identité de la personne qui le possède. Lorsque la continuité mémorielle échoue à procurer à l'individu ses conditions de persistance dans le temps, certaines propriétés sociales, du fait de leur caractère durable, peuvent les lui conférer.

\section{ACTION, INTENTION ET IDENTITÉ PERSONNELLE}

Toute action comporte au moins trois phases. Elle présuppose tout d'abord la formation de préférences de la part de l'acteur, c'est-à-dire de croyances portant sur l'objet de l'action. Ces croyances donnent lieu à une intention, qui concerne la façon dont l'individu souhaite intervenir dans le monde qui l'entoure. Une seconde phase consiste en la mobilisation de moyens en vue de la réalisation de l'objectif. C'est ici qu'apparaissent les capacités instrumentales de l'acteur. Lorsque les moyens mis à la disposition de l'action sont adéquats, l'individu peut être considéré comme rationnel. La troisième phase est son exécution. Sur la base de l'intention et des moyens mobilisés pour la réaliser, l'individu accomplit l'action. Des facteurs comme la volonté ou la concentration peuvent être déterminants à ce stade.

Cette caractérisation élémentaire de l'action est très intéressante du point de vue de la théorie de l'identité personnelle. Elle démontre, d'une part, que toute action présuppose l'identité de l'individu qui l'effectue. En d'autres termes, il n'est d'action qui ne soit accomplie par une personne. D'autre part, elle témoigne du fait que l'accomplissement d'une action renforce l'identité personnelle, en consolidant le rapport existant entre les différentes «phases» temporelles de l'individu.

Remarquons, en premier lieu, que toute action s'inscrit dans la durée. Entre le moment où je forme mes préférences et celui où j'agis, un certain laps de temps 
s'est nécessairement écoulé. En second lieu, l'accomplissement d'une action présuppose la persistance dans le temps des états mentaux de l'acteur. Pour qu'une action soit menée à bien, les croyances de l'individu doivent former un arrièreplan stable tout au long de cette dernière. Imaginons ce qui se passerait si, entre le moment où j'ai formé une intention et celui où je mobilise les moyens à ma disposition, je changeais de croyances concernant le caractère désirable de mon action. Il est évident que cette dernière n'aurait pas lieu. Elle serait interrompue du fait même de la disparition des croyances qui l'ont motivée.

La persistance des états mentaux de l'acteur peut servir de «socle» à son identité personnelle. Le noyau permanent de croyances et d'intentions nécessaire à l'accomplissement d'une action fournit à l'individu ses conditions de subsistance dans le temps. Dans un article particulièrement intéressant, Joëlle Proust envisage la relation existant entre l'identité personnelle et les pathologies de l'action ${ }^{26}$. Elle identifie plusieurs cas de comportements défectueux ayant pour origine la disparition, au cours de l'action, des états mentaux qui se trouvaient à son fondement. Par exemple, un individu atteint de «confabulation» change si rapidement de préférences qu'il en devient incapable d'accomplir une action de part en part. Il n'a de cesse de commencer et d'abandonner en cours de route ses comportements, ce qui rend ces derniers incompréhensibles pour autrui.

Selon Proust, les pathologies de l'action conduisent dans de nombreux cas à des troubles de la personne. Examinant une expérience relatée par Oliver Sacks, elle affirme qu' «Un cas très sévère d'amnésie avec confabulation (...) peut conduire à conclure, comme le fait l'auteur, que le patient privé du récit de la continuité de sa vie, a perdu avec son histoire le soubassement de sa personne.» ${ }^{27}$ Par «récit de la continuité de sa vie», il faut comprendre l'ensemble des croyances, des désirs et des intentions qui se trouvent au fondement de l'individu. Ce récit peut porter sur des faits plus ou moins éloignés dans le passé. Une action peut être incohérente parce que l'individu qui l'accomplit ne se souvient pas des croyances qu'il avait adoptées quelques instants plus tôt. Mais elle peut l'être aussi parce qu'il a perdu contact avec des informations plus générales le concernant, comme son nom, son métier ou la nature de son entourage.

Les sujets normaux se comportent la plupart du temps de façon cohérente. L'interruption d'une action est, chez eux, l'exception plutôt que la règle. Pourquoi? Parce qu'un individu normal parvient à conserver ses intentions et ses croyances tout au long de la planification de ses comportements. Contrairement au sujet pathologique, il maintient le «soubassement de sa personne» en gardant intact le fil de son «histoire», c'est-à-dire le fil de ses états mentaux. La capacité à mener à bien une action présuppose donc l'existence d'une identité personnelle, laquelle consiste en la pérennité des croyances qui motivent le comportement. Bien plus, il semble que l'accomplissement d'une action renforce l'identité de l'acteur. Le fait, pour ce dernier, de pouvoir jeter un regard rétrospectif sur ses comportements et de constater que c'est lui qui les a accomplis affermit en lui le sentiment de sa propre identité. Comme le dit Joëlle Proust, «Pour un sujet nor-

Joëlle Proust, «Identité personnelle et pathologie de l'action», in Isaac Joseph et Joëlle Proust (éds.), 1996, La folie dans la place, Paris, EHESS.

27 Ibid., p. 162. 
mal, l'intention produite par (...) A préside à l'action de A. La continuité psychologique entre les étapes intention-causation-action (...) permet à A de réidentifier comme lui-même l'élément intentionnel causal qui organise et maintient l'orientation de la séquence jusqu' à son achèvement.» ${ }^{28} \mathrm{Il}$ faut donc admettre que l'identité personnelle et les actions entretiennent un rapport d'influence mutuelle. La réalisation d'une action présuppose formellement l'identité de l'individu, c'est-àdire sa permanence dans le temps. A l'inverse, cette dernière se consolide sous l'effet de la capacité de «réidentification» dont fait preuve l'acteur au cours de son action.

Nous n'avons envisagé jusqu'à présent que les actions en général. Est-il possible de préciser le lien existant entre l'identité personnelle et les actions sociales? L'action sociale est définie classiquement par Max Weber comme «(...) l'activité qui d'après son sens visé par l'agent ou les agents, se rapporte au comportement $d$ 'autrui, par rapport auquel s' oriente son déroulement. ${ }^{29}$ Elle a en commun avec toutes les formes d'action le fait que celui qui l'accomplit lui confère un «sens subjectif », c'est-à-dire, dans le langage sociologique contemporain, dispose de raisons de l'effectuer. Une action est considérée comme sociale lorsque son sens subjectif découle de l'influence qu'exercent sur elle les comportements d'autres individus.

Dans le cas d'une action sociale, le comportement de l'acteur repose non seulement sur ses propres états mentaux, mais aussi sur les états mentaux d'autrui. Je me comporte d'une certaine manière, d'une part, parce que mes croyances et mes intentions me conduisent à le faire. D'autre part, j'accomplis une action parce que les croyances et les intentions d'autres individus sont de telle ou telle sorte. On peut donc soutenir que toute action sociale implique une relation «dialectique» entre plusieurs ensembles d'états mentaux.

Comme nous l'avons montré, la mise en œuvre de toute action implique la pérennité temporelle des croyances de l'acteur. Cette pérennité contribue à lui conférer une identité personnelle, définie comme le noyau immuable de croyances et d'intentions nécessaire au passage à l'action. Or, il semble que la réalisation d'une action sociale implique non seulement la pérennité des états mentaux de l'acteur, mais aussi la pérennité des états mentaux des individus dont la présence oriente l'action. Pour qu'une action sociale soit accomplie, il faut, d'une part, que l'acteur perçoive les états mentaux des individus qui orientent son action comme doués d'une certaine cohérence, et, d'autre part, que cette cohérence s'inscrive dans la durée, sans quoi l'individu ne pourrait pas orienter son comportement par rapport à leurs croyances.

Si la capacité à agir en général constitue un « socle» potentiel de l'identité personnelle, la capacité à agir socialement l'est, par conséquent, de façon privilégiée. Pour que $\mathrm{X}$ oriente son action par rapport à celle de $\mathrm{Y}$, il faut qu'il comprenne cette dernière. Pour que l'action de Y soit intelligible, il est nécessaire que ses états mentaux soient cohérents, c'est-à-dire dotés d'une certaine continuité dans le temps. Dans le cadre d'une action sociale, l'identité d'une personne bénéficie par conséquent d'un double appui: ses propres états mentaux et les états mentaux de

\footnotetext{
$28 \quad$ Ibid., p. 170.

29 Max Weber, 1995, Economie et société, tome I, Paris, Plon, p. 28.
} 
celui ou ceux par rapport auxquels il oriente son action. On peut donc en conclure que la capacité des individus à accomplir des actions sociales influe sur la persistance dans le temps de leur identité personnelle.

\section{Institut des sciences humaines appliquées}

Université de Paris IV-Sorbonne

\section{RÉFÉRENCES BIBLIOGRAPHIQUES}

S. Beaud et M. Pialoux, 1999, Retour sur la condition ouvrière. Enquête dans les usines Peugeot de Sochaux-Montbéliard, Paris, Fayard.

R. Boudon, 1979, La logique du social, Paris, Hachette.

R. Boudon,1986, L'idéologie, ou l'origine des idées reçues, Paris, Fayard.

R. Boudon, 1997, «Le "paradoxe du vote" et la théorie de la rationalité», in Revue française de sociologie, vol. 38 .

R. Boudon et François Bourricaud (éds.), 1982, Dictionnaire critique de la sociologie, Paris, PUF.

R. Dawkins, 1978, Le gène égoïste, Paris, Mengès.

D. Dennett, 1990, La stratégie de l'interprète, Paris, Gallimard, coll.

J. Elster (éd.), 1986, The Multiple Self, Cambridge, Cambridge University Press.

S. Ferret (éd.), 1998, L'identité, Paris, Flammarion.

B. Garrett, 1998, Personal Identity and Self-Consciousness, Londres et New York, Routledge.

I. Hacking, 2000, The Social Construction of What?, Cambridge, Harvard University Press.

A. Kardiner, 1969, L'individu dans sa société, Paris, Gallimard.

R. Merton, 1976, Sociological ambivalence and other essays, New York et Londres, The Free Press.

A. Oksenberg Rorty (éd.), 1976, The Identities of Persons, Berkeley, University of California Press.

D. Parfit, 1984, Reasons and Persons, Cambridge, Cambridge University Press.

J. Perry (éd.), 1975, Personal Identity, Berkeley, University of California Press.

J. Proust, 1996, «Identité personnelle et pathologie de l'action», in I. Joseph et J. Proust (éds.), La folie dans la place, Paris, EHESS.

W. v. O. Quine, 1977, Le mot et la chose, Paris, Flammarion.

J.-Cl. Ruano-Borbalan (éd.), 1998, L'identité, Paris, Ed. Sciences Humaines.

M. Weber, 1995, Economie et société, tome I, Paris, Plon. 